\title{
A SIMPLE METHOD FOR THE ASEPTIC FILLING OF SCREW-CAPPED CONTAINERS
}

\author{
BY \\ J. E. O'HAGAN \\ From the Laboratories of the Red Cross Blood Transfusion Service, Brisbane, Australia
}

(RECEIVED FOR PUBLICATION FEBRUARY 5, 1952)

The increasing use of screw-capped tubes and bottles in bacteriology, following their introduction by McCartney (McCartney, 1933; Mackie and McCartney, 1948), suggests many applications for a method which has been used successfully for dispensing pooled human serum for transfusion. The principle of the method adopted is that the glass container is autoclaved with its cap fitting loosely, but suitably wrapped, the wrappings are removed and the cap pushed into a "gripper" under a hood, while the container is filled under the same hood by transferring its neck to a spring-loaded valve which delivers sterile fluid. The " gripper," a hard rubber ring with a tapered inner hole, is used both to remove and to replace the cap. On filling to the desired level, the valve is shut by lowering the container, which is then screwed up into the firmly held cap. Should it be desired to keep the outside of the cap sterile, this can be achieved by covering the cap with sterile paper before giving a final tightening by hand.

A description of the technique for filling halflitre serum bottles will illustrate the method.

\section{The Apparatus}

The hood is constructed as follows (Fig. 1). A 108-oz. clear glass Winchester is cut 10 in. from the top by means of a hot wire glass cutter and the edge ground on a sheet of flat iron fed with a paste of 80 mesh carborundum in $50 \%$ aqueous glycerine. A $5 \frac{1}{4}$ in. circle of $\frac{1}{2}$ in. thick firm rubber (B) is then ground on an emery wheel to fit snugly into the neck of $A$. On the underside of B a sheet of $16 \mathrm{G}$. stainless steel or brass (C) is mounted to give rigidity, and then the cap gripper (M) of firm rubber ( 3 in. diameter, $\frac{7}{8}$ in. thick) is attached with four 1-in. wood screws (R). One side of the gripper is ground to allow the valve to operate smoothly with this size bottle and a recess made also to allow the 5 -in. carriage bolt (D, with its round head ground flat) to seat properly.

The valve is built in the following way. To a $1 \frac{1}{2}$ in. diameter stainless steel or brass disc $(Q)$ with a $\frac{1}{2}$ in. diameter central hole are soldered two stainless steel or phosphor bronze rods on one side, and on the other a rubber washer is secured by means of two small aluminium rivets. This washer may be made in the same mould as the rubber closure which fits inside the cap, but should be harder. The rods pass through bearings made from two worn $11 \mathrm{G}$. transfusion needles $(\mathrm{J})$ cut off to within $\frac{1}{2}$ in. of the hub and joined by a brass bar at the top. Then, after a $10 \mathrm{~mm}$. glass tube $(\mathrm{H})$ and a suitable spring (I) have been fitted, the rods are softsoldered to a brass bar at $K$. A length of 3/16 in. I.D. gum rubber tubing $(G)$ is fixed to $H$ and coiled to fit through the valve assembly, two screw-eyes (not shown) with $\frac{1}{2}$ in. diameter holes being fixed immediately in front of and behind the valve to guide the rubber through. The assembly is then placed in the hood, the rubber tubing $(G)$ attached to the $8 \mathrm{~mm}$. glass tube (F) which, with the carriage bolt (D), is placed through holes in the rubber stopper (E). The brass collar $(O)$ is then fixed and the bolt secured with a washer and nut. A removable spacer bar (L) is inserted through $\frac{1}{2}$ in. holes in $B$ and $C$ to hold the valve mechanism open, and so prevent the rubber tubing from sticking during autoclaving.

The hood with spacer bar in position is rinsed, placed on one layer of lint and two of brown paper, and these are then tied firmly at the neck. The upper end of $F$ is connected by rubber tubing to the large vessel intended to hold the sterile fluid. The vessel and its attachments are then autoclaved, and, after filling the vessel by Seitz filtration, they are conveyed to a "sterile" room, in which is arranged a shelf to hold the vessel, a fixed stand with a suitable clamp to hold the hood by the neck, and a pivoted adjustable rest on which the base of the bottle sits during the filling.

The receiving bottles are prepared, after suitable washing treatment, by rinsing with pyrogen-free distilled water, and, after draining, $3 \mathrm{ml}$. of similar water is run in. The caps may require slight crimping below the knurling if a large soft rubber closure is to be retained. Each cap, with the rubber closure in position, is then placed loosely on the top of a bottle, turned anticlockwise until it drops as far down as it will go, and then turned a further quarter turn. The caps are covered 


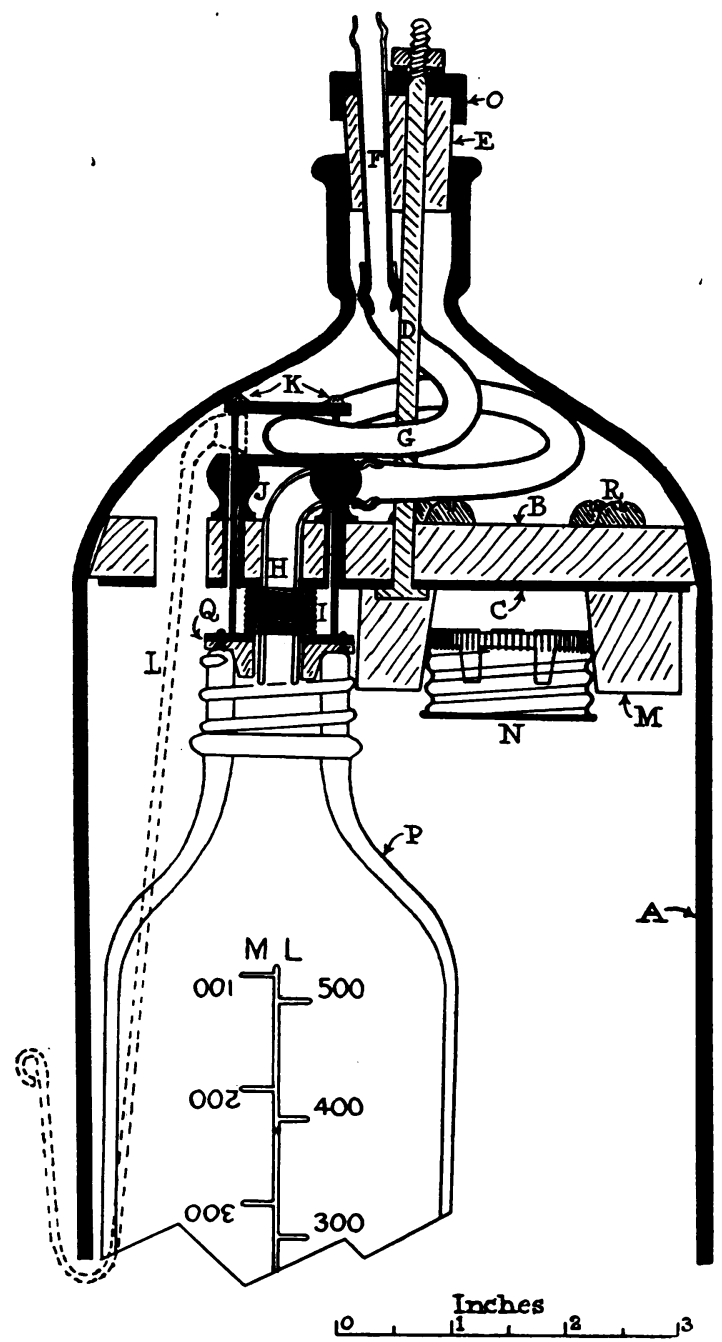

FIG. 1.-A. Top of glass Winchester. B. $\frac{1}{2}$ in thick rubber sheet C. Stainless steel sheet 16 G. D. 5 in. carriage bolt. E. Rubber stopper. F. Glass inlet tube $8 \mathrm{~mm}$. O.D. G. $3 / 16$ in. I.D. amber rubber tube. H. Glass delivery tube $10 \mathrm{~mm}$. O.D. I. Spring. J. Old 11 G. needles. K. Brass bar, soft soldered. L. Spacer bar. M. Rubber "gripper." N. Aluminium cap with rubber closure in place and holes in top for insertion of needles. $O$. Metal collar. P. Pyrex bottle in filling position. Q. Stainless steel or brass disc. R. Wood screws holding "gripper."

with a layer of lint and two layers of brown paper, which are held in place by means of a strong rubber band placed well down on the neck. The bottles are then autoclaved at $20 \mathrm{lb}$. per square inch for 20 minutes. Complete drying of the bottle interior is not necessary, since the neck and cap are the last parts to cool (due to the wrappings) and moisture does not condense there.

\section{Filling Procedure}

The bottles are then transferred to the "sterile" room. The hood is clamped in position and uncovered. The wrappings of a bottle are removed, without disturb- ing the cap, by raising an edge with the thumb and forefinger of the right hand while the middle finger rests on the top and the other fingers on the rear of the neck of the bottle (Fig. 2). The cap is pushed up into the "gripper" (Fig. 3) and the bottle transferred to the valve; in the case of the first bottle, the spacer bar is removed and the bottle rest adjusted to the correct height (Fig. 4). Air pressure is then applied to the storage vessel to deliver its contents.

After filling to the desired level the bottle is lowered slightly to cut off the flow and inserted once again in the cap (Fig. 5), into which it is screwed up using both hands on the base of the bottle. A feature of the device is that when the spring (I) expands, the tip of the delivery tube $(\mathrm{H})$ is covered and it is practically impossible for the operator to wet the rim of the bottle and so leave a trail for the entry of micro-organisms. By using a soft rubber closure of the type shown inserted in $\mathbf{N}$, a tight dry seal is maintained at all times. The sloping projections should not be too long, however, or the closure will be drawn down on to the bottle during autoclaving.

If the top of the cap is to be kept sterile it is covered with a sterile double-folded sheet of brown paper, a rubber band placed to hold it, and the cap given an extra tightening by hand. Later the paper may be removed and the cap dipped into a plastic capping solution* to which phenol has been added to a concentration of $4 \%$.

Small volumes of the sterile fluid may be run off at any stage for routine sterility, toxicity, biochemical, or other tests by pushing the lip of a sterile test-tube on the valve. If screw-topped test-tubes $\dagger$ are used and a suitable gripper provided under the hood, "flaming" may be dispensed with altogether, but if cotton-plugged tubes are used it is well to see that the rim is well covered and flamed before and after insertion under the hood.

To clean the hood the spacer bar is replaced and the rubber inlet tube placed on a water jet, followed by rinsing with pyrogen-free water. After using three times, the inner rubber tube $(G)$ should be removed and thoroughly cleaned, being replaced after using six times. Grippers may need occasional replacement. Worn grippers may have a small segment cut from them and are then useful for removing tight caps from used bottles.

\section{Results}

At the Blood Transfusion Service laboratories of the Queensland Division of the Australian Red Cross Society over 500 batches, comprising more than 5,000 litres of pooled human serum, have been bottled without one reported contamination. Seitzfiltered media have been filled also by this means and given negative sterility tests. One operator, unaided, can fill 60 half-litre bottles per hour.

* N/F capping solution supplied by the London Capsule Company. + Culture tubes No. $45066.20 \times 150 \mathrm{~mm}$. made by the Kimble Glass Company. 




FIG. 2.-Removing coverings.

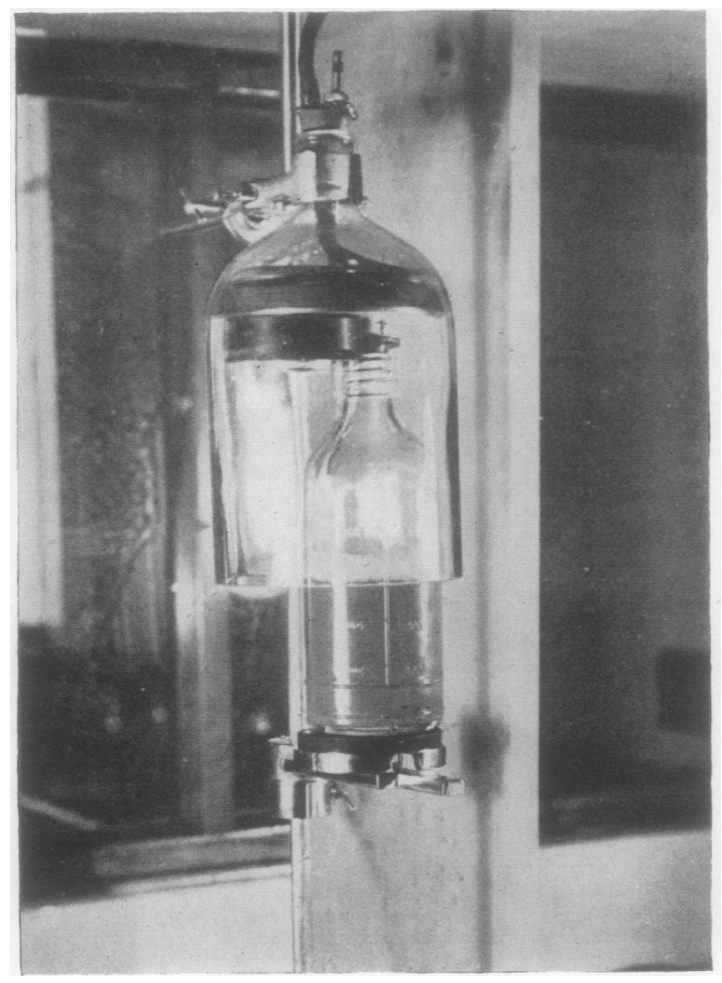

FIG. 4.-Filling.

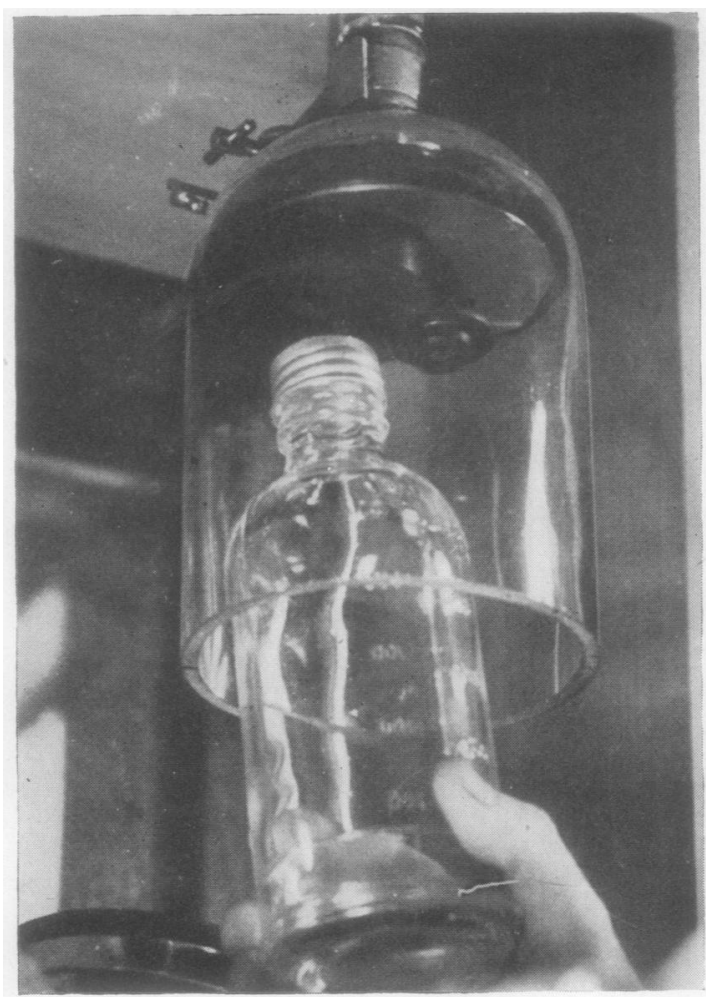

FIG. 3.-Removing cap with " gripper."



FIG. 5.-Screwing bottle into cap. 


\section{Summary}

The construction of a device and the technique of a method for the aseptic filling of screw-capped containers is described.

The method is simple, rapid, and convenient, and the conventional flaming procedures may be dispensed with entirely.

No contaminations were reported following the filling of more than 5,000 litres of pooled human serum for transfusion.
I am much indebted to Dr. A. E. Shaw for his continuous encouragement and helpful advice; to Mr. A. Tuffley for the photographs; to the Queensland Rubber Co. for the rubber mouldings; and to Misses H. Crawford and M. Tunn and Mr. M. Robins for the sterility checks.

\section{REFERENCES}

McCartney, J. E. (1933). Lancet, 2, 433.

Mackie, T. J., and McCartney, J. E. (1948). Handbook of Practical Bacteriology, 8th ed., p. 141. Edinburgh. 